\title{
Myxofibrosarcoma of the maxillary sinus
}

\author{
Anni Wong, B.A., M.S., ${ }^{1}$ Richard Chan Woo Park, M.D., F.A.C.S., ${ }^{1}$ Neena M. Mirani, M.D., ${ }^{1-3}$ \\ and Jean Anderson Eloy, M.D., F.A.C.S. ${ }^{1,3-5}$
}

\begin{abstract}
Background: Myxofibrosarcoma (MFS) is a common sarcoma in the extremities of older individuals but is extremely uncommon in the head and neck region. Diagnosis may be challenging but is critical to the management of the patient. We discuss the radiographic and histopathologic characteristics of this destructive tumor. The distinguishing features of MFS and its differential diagnosis are reviewed to familiarize the managing otolaryngologist with this rare entity.

Methods: A 61-year-old woman presents with a 6-week history of severe left facial pain and left eye pain. Imaging demonstrates significant right and left-sided maxillary sinus opacification with destruction of the left maxillary sinus as well as the left medial and inferior orbital walls.

Results: Histopathologic examination revealed spindle and stellate tumor cells of variable cellularity in myxoid stroma with cellular pleomorphism consistent with MFS of intermediate-to high grade. The patient underwent resection of the left-sided lesion with orbital exenteration and repair of the defect with microvascular free flap followed by postoperative radiotherapy.

Conclusion: MFS must be differentiated from other lesions with myxoid qualities. Histopathologic examination is required for definitive diagnosis. Management includes complete tumor excision with adequate tumor margins. Adjuvant postoperative radiotherapy should be considered for larger tumors with positive resection margins or lesions of intermediate-to-high grade.
\end{abstract}

(Allergy Rhinol 8:e95-e99, 2017; doi: 10.2500/ar.2017.8.0200)

$\mathbf{M}$ yxofibrosarcoma (MFS) is a rare histologic subtype of soft-tissue sarcomas of fibroblastic origin. Although MFS is a common sarcoma in the extremities, trunk, and retroperitoneum of elderly patients between the sixth and eighth decade of life, it is rare in the head and neck region. ${ }^{1,2}$ Sarcomas of the head and neck account for $<1 \%$ of head and neck malignancies, with only a few cases of MFS reported in this region. ${ }^{3}$ To our knowledge, there have only been three reported cases of MFS in the maxillary sinus, with this being the fourth. ${ }^{4-6}$ These rare neoplasms can be difficult to diagnose clinically, radiologically, and histologically. Magnetic resonance imaging (MRI) and

From the ${ }^{1}$ Department of Otolaryngology_Head and Neck Surgery, Rutgers New Jersey Medical School, Newark, New Jersey, ${ }^{2}$ Department of Pathology, Rutgers New Jersey Medical School, Newark, New Jersey, ${ }^{3}$ Department of Ophthalmology and Visual Science, Rutgers New Jersey Medical School, Newark, New Jersey, ${ }^{4}$ Center for Skull Base and Pituitary Surgery, Neurological Institute of New Jersey, Rutgers New Jersey Medical School, Newark, New Jersey, and ${ }^{5}$ Department of Neurological Surgery, Rutgers New Jersey Medical School, Newark, New Jersey

No external funding sources reported

The authors have no conflicts of interest to declare pertaining to this article Address correspondence to Jean Anderson Eloy, M.D., Endoscopic Skull Base Surgery Program, Department of Otolaryngology-Head and Neck Surgery, Neurological Institute of New Jersey, Rutgers New Jersey Medical School, 90 Bergen St., Suite 8100, Newark, NJ 07103

E-mail address: jean.anderson.eloy@gmail.com

This work is published and licensed by OceanSide Publications, Inc. The full terms of this license are available at https://www.allergyandrhinology.com/terms and incorporate the Creative Commons License Deed: Attribution - Non-Commercial - NoDerivs 4.0 Unported (CC BY-NC-ND 4.0). By accessing the work you hereby accept the terms. Non-commercial uses of the work are permitted without any further permission from OceanSide Publications, Inc., provided the work is properly attributed. Any use of the work other then as authorized under this license or copyright law is prohibited. Copyright (C) 2017, OceanSide Publications, Inc., U.S.A. computerized tomographies (CT) may show malignant features, but histopathologic examination is required for a definitive diagnosis. We presented a case of an aggressive intermediate-to-high-grade MFS of the maxillary sinus.

\section{CASE REPORT}

A 61-year-old woman was referred for evaluation of bilateral sinus disease and left eye proptosis, with a 6-week history of progressively worsening severe left facial pain and pressure. She also had left eye pain, blurry vision, and left facial numbness. Her medical history was significant for hypercholesterolemia, hypertension, a history of thyroid surgery, and a 13-packyear smoking history. Results of her physical examination were significant for left-sided hypoglobus with some strabismus. The rest of her head and neck examination was unremarkable. There was no cervical lymphadenopathy or evidence of any neck mass. A CT showed significant right- and left-sided maxillary sinus opacification, with destruction of the lateral and inferior floor of the left maxillary sinus as well as the left medial and inferior orbital walls. MRI of the orbits and paranasal sinuses demonstrated a left maxillary sinus mass with intraorbital extension suggestive of a maxillary sinus mucocele (Fig. 1). Right maxillary opacification with expansion of the medial maxillary sinus wall was also noted.

The patient underwent bilateral maxillary antrostomy with biopsy. On the right side, the histopathologic examination was consistent with a mucocele. Histopathologic examination of the left-sided lesion 

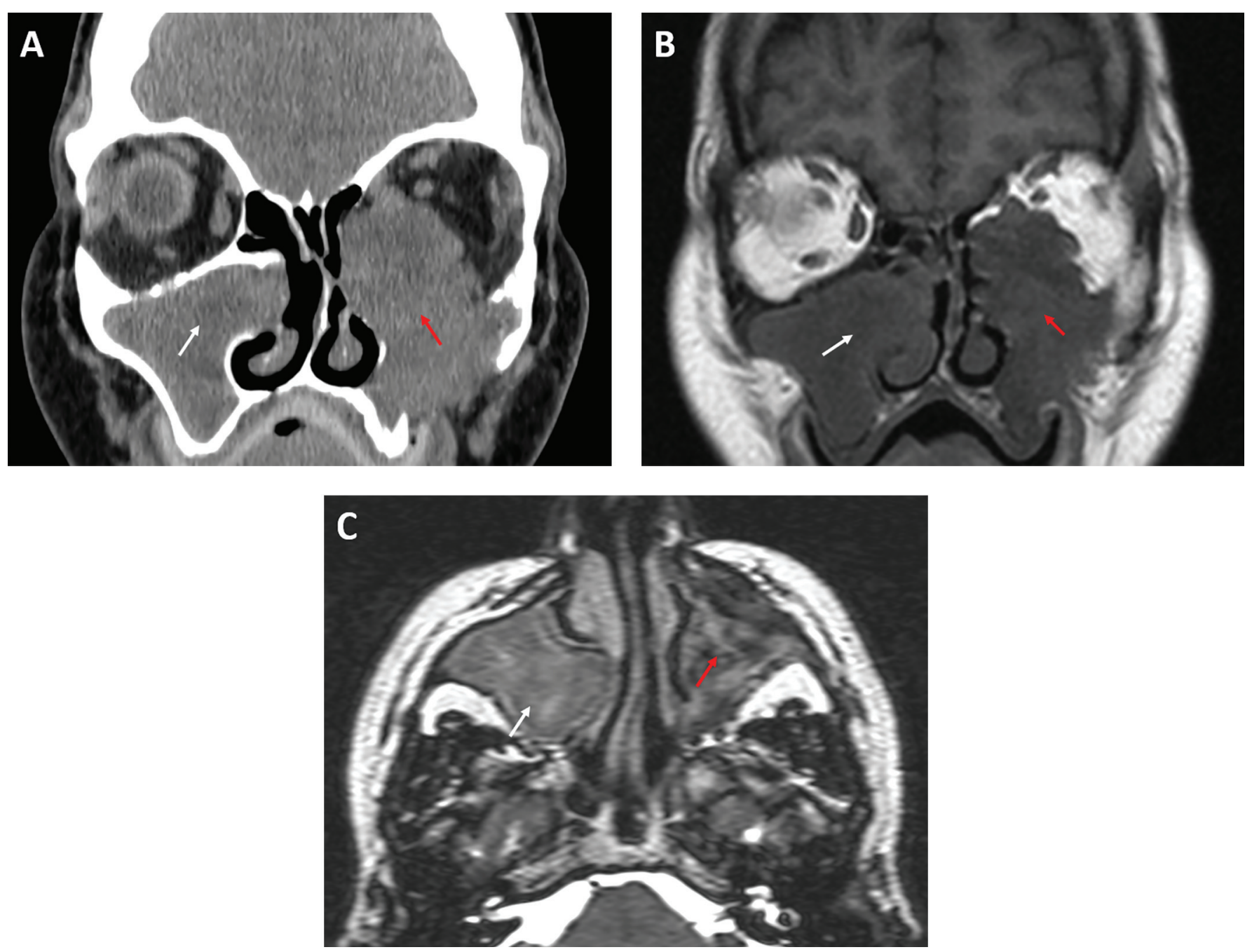

Figure 1. (A) Coronal computed tomography of the paranasal sinuses reveals an expansile left sinonasal mass (red arrow) centered in the maxillary sinus with destruction of the lateral nasal wall and the medial and inferior orbital walls; there also is right-sided maxillary sinus opacification (white arrow), with expansion of the medial maxillary wall. (B) Coronal T1-weighted magnetic resonance image (MRI) of the paranasal sinuses, showing a large left hypointense lesions (red arrow) in the maxillary sinus, with marked intraorbital extension; there is a right hypointense lesion (white arrow), with expansion of the medial orbital wall. (C) On axial T2-weighted MRI, a left slightly hyperintense lesion with heterogenous contents (red arrow) can be seen; on the right, a similar hyperintense lesion is noted (white arrow) with less heterogeneity.

revealed spindle and stellate tumor cells of variable cellularity in myxoid stroma with cellular pleomorphism consistent with MFS of intermediate-to-high grade (Fig. 2). Immunohistochemical stains of the tumor cells were positive for vimentin (Fig. 2 F), partially positive for smooth-muscle antigen (SMA), negative for desmin and S100, a protein used as a marker for epidermal differentiation. A positron emission tomography/CT was obtained, which did not show significant metastatic disease. Our patient underwent combined open and endoscopic resection of the left-sided lesion with orbital exenteration, and repair of the defect with a microvascular free flap followed by postoperative radiotherapy.

\section{DISCUSSION}

MFS is a fibroblast-derived soft-tissue sarcoma that is frequently found in the extremities and trunk of elderly individuals but rarely encountered in the head and neck region. ${ }^{1,2}$ MFS was known as a myxoid variant of malignant fibrous histiocytoma but was declassified as such by the World Health Organization in 2002 and is now recognized as a distinct pathologic entity. Malignant fibrous histiocytomas that are predominantly myxoid (>50\%), without myogenic, lipoblastic, and chondrogenic features rendered a separate diagnosis of MFS. ${ }^{2,6-8}$ The clinical presentation of MFS in the sinonasal tract does not distinguish itself from other soft-tissue neoplasms because they all similarly 


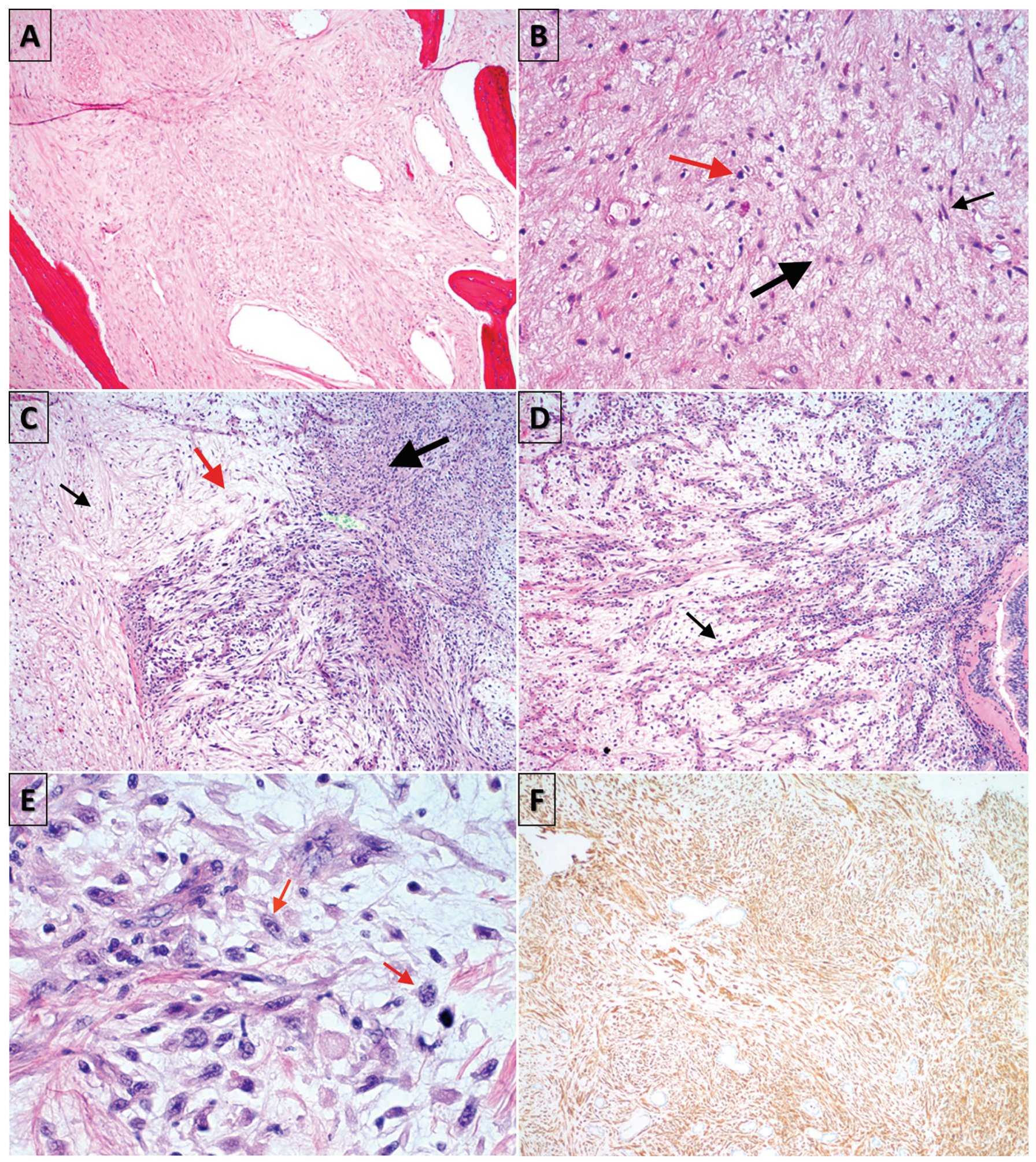

Figure 2. Histopathologic examination, demonstrating (A) hypocellular tumor in maxillary bone (hematoxylin and eosin [HEE], original magnification $\times 40 ;(B)$ spindle (black arrow) and stellate cells (thick black arrow), with hyperchromatic nuclei (red arrow) in a myxoid matrix (HEE, original magnification $\times 100)$; (C) alternating hypocellular (black arrow), myxoid (red arrow), and hypercellular (thick black arrow) fibrous areas (HEE, original magnification $\times 100) ;(D)$ curvilinear, thin-walled blood vessels (black arrow) prominent in myxoid areas (HEE, original magnification $\times 100)$; (E) nuclear pleomorphism and mitosis (red arrows) $($ HEE, original magnification $\times 400)$; and $(F)$ immunologic staining positive for vimentin, an intermediate filament protein, confirming mesenchymal origin of fibroblasts at (original magnification $\times 100)$. 
present with epistaxis, nasal obstruction, rhinorrhea, and nonspecific nasal discomfort. ${ }^{5}$ The diagnosis of MFS is based on characteristic histopathologic features, including the (1) presence of alternating hypocellular, myxoid areas, and hypercellular, fibrous areas; (2) pleomorphic nuclei; (3) curvilinear, thin-walled blood vessels prominent in myxoid areas; (4) aggregation of neoplastic cells or inflammatory cells; and (5) spindle and stellate cells in myxoid matrix. ${ }^{2,9}$ Immunologic staining is typically positive for vimentin and CD-34, which indicates the tumor's fibroblastic origin, sometimes positive for SMA and negative for S-100 protein. 4,9

MRIs and CTs may show malignant features, e.g., local invasion, and may help with diagnosis but are not diagnostic. Low-grade MFS on CT is often misinterpreted as a benign tumor, due to its presentation of a relatively smooth surface, uniform density, and distinct separation of tumor from the surrounding soft tissues. ${ }^{10}$ On contrast-enhanced CT, low-grade MFS typically enhances more than muscle. ${ }^{11}$ Sarcomas with high myxoid content, such as MFS, may appear misleadingly cystic on MRI, described by hyperintense T2-weighted signals owing to the high intracellular water content of the myxoid cells. ${ }^{12}$ Awareness and suspicion of MFS in this anatomic area should remain high to avoid erroneous identification of this covert malignancy as a benign cystic lesion such as a mucocele, as evident in our case.

A review of multiple MRI sequences is helpful in differentiating between MFS and mucoceles because the latter lesion can transition from hypo- to hyperintensity on T1-weighted MRI due to chronic inspissation over time, ${ }^{13}$ whereas MFS remain hypointense. In contrast to mucoceles, MFS display an infiltrative pattern on T2-weighted MRI. This infiltrative pattern can be further elucidated with detail with gadolinium-enhancement on T1-weighted MRI, which can demonstrate abnormal multidirectional signal extensions along the fascial plane. ${ }^{14}$ It is important to distinguish MFS from other benign or malignant tumors with myxoid qualities because its differences can be subtle, and the required treatment and follow-up may vary. A differential diagnosis includes low-grade fibromyxoid sarcoma (LGFS), myofibroblastic sarcoma, myxoid neurofibroma, myxoid liposarcoma, and benign myxomatous tumors. ${ }^{9}$

Myxoid liposarcoma can be differentiated from MFS by the presence of scattered lipoblasts and its fine plexiform vascular pattern compared with the curvilinear capillary pattern seen in MFS. ${ }^{8}$ Neurofibromas present with more elongated wavy nuclei and are extensively positive for S-100. ${ }^{12}$ Myofibroblastic sarcomas also present with spindle cells in a variable amount of myxoid matrix but are diagnostically positive for SMA and demonstrate myofilaments that ex- tend to the cell surface.$^{15}$ Distinction between MFS and LGFS is probably the most challenging. Although both MFS and LGFS are composed of alternating myxoid and fibrous stroma with spindle cells, the spindle cells in LGFS fall in a linear arrangement, which creates a swirling pattern. In addition, MFS feature more cytologic atypia and nuclear pleomorphism than LGFS. ${ }^{12}$ LGFS ultimately become malignant, with metastasis in $>50 \%$ of the cases, whereas low-grade MFS are rarely metastatic, ${ }^{10}$ which underlines the importance of distinguishing between these two tumors.

Tumors are graded on a three- or four-tier system, based on the degree of cellularity (proportion of hypocellular myxoid versus cellular areas), mitotic activity, and nuclear pleomorphism. ${ }^{5,6}$ Low-grade MFS predominantly consists of hypocellular myxoid matrix. ${ }^{6,10}$ Low-grade tumors have low malignancy potential and rarely metastasize, which indicates a good short-term prognosis, with 5- and 10-year survival rates at 65 and $52 \%$, respectively. ${ }^{9,10}$ However, the local recurrence rate for low-grade MFS is as high as that of highergrade tumors, at $\sim 50$ to $61 \%$. $^{2,6,10}$ Recurrence of lowgrade MFS generally occurs with progression to higher histologic grades in 15-38\% of cases, which acquires increasing metastatic potential. ${ }^{2}$ In a study by Sanfilippo et al., ${ }^{16}$ it was reported that the quality of surgical margins predicted local recurrences, whereas histologic grade determined the risk of metastases.

Therefore, a proper diagnosis followed by complete surgical resection with adequate resection margins with a goal of $1-3 \mathrm{~cm}$ is critical for disease cure. ${ }^{10}$ Sufficient wide surgical resections may be challenging to achieve within the delicate head and neck region when considering its strict anatomic constraints, unlike the trunk or extremities where sarcomas are usually found. ${ }^{17}$ Adjuvant postoperative radiotherapy is recommended in cases with close $(<1 \mathrm{~cm})$ negative surgical margins and large or high-grade MFS to avoid local and distant recurrences., ${ }^{6,17}$ The role of chemotherapy is currently debatable because results have been relatively poor. ${ }^{5,10}$ Due to nonspecific clinical presentation and the rarity of these tumors in the head and neck region, definitive preoperative diagnosis can be difficult. Histologic examination is required for a diagnosis. Although uncommon, this present case demonstrated that MFS should be considered as a differential diagnosis when a myxoid tumor is encountered because an accurate diagnosis is crucial to the clinical course and outcome of the patient. Initial aggressive local management, followed by close surveillance is imperative because MFS tend to have a high recurrence rate.

\section{CONCLUSION}

This is a rare case of MFS that arose in the maxillary sinus with extension into the orbit. Diagnosis may be 
challenging but is critical to the management of the patient. Complete tumor excision with adequate tumor margins serves as the criterion standard of treatment. Adjuvant postoperative radiotherapy should be considered for lesions of intermediate-to-high grade or larger tumors with positive resection margins. The extent, rarity, and aggressive nature of the disease, coupled with the complexity of the head and neck region emphasized the need for an interdisciplinary approach to reach a proper diagnosis and provide appropriate management for the patient.

\section{REFERENCES}

1. Gokavarapu S, Mustyala B, Kumar J, et al. Large myxofibrosarcoma of oral cavity: A rare clinical presentation in lower gingivobuccal sulcus. Oral Sci Int 12:27-30, 2015.

2. Haglund KE, Raut CP, Nascimento AF, et al. Recurrence patterns and survival for patients with intermediate- and highgrade myxofibrosarcoma. Int J Radiat Oncol Biol Phys 82:361367, 2012.

3. Nishimura G, Sano D, Hanashi M, et al. Myxofibrosarcoma of the hypopharynx. Auris Nasus Larynx 33:93-96, 2006.

4. Qiubei Z, Cheng L, Yaping X, et al. Myxofibrosarcoma of the sinus piriformis: Case report and literature review. World J Surg Oncol 10:245, 2012.

5. Lam PK, Trendell-Smith N, Li JH, et al. Myxofibrosarcoma of the sphenoid sinus. J Laryngol Otol 116:464-466, 2002.

6. Dell'Aversana Orabona G, Iaconetta G, Abbate V, et al. Head and neck myxofibrosarcoma: A case report and review of the literature. J Med Case Rep 8:468, 2014.
7. Jo VY, and Fletcher CD. WHO classification of soft tissue tumours: An update based on the 2013 (4th) edition. Pathology 46:95-104, 2014.

8. Wang M, Khurana RN, Parikh JG, et al. Myxofibrosarcoma of the orbit: An underrecognized entity? Case report and review of the literature. Ophthalmology 115:1237-1240.e2, 2008.

9. Gugatschka M, Beham A, Stammberger H, et al. First case of a myxofibrosarcoma of the vocal folds: Case report and review of the literature. J Voice 24:374-376, 2010.

10. Taghi AS, Ali A, Kuchai R, and Saleh H. Rare sarcoma presented as sinusitis. BMJ Case Rep 2012: pii: bcr0220125863, 2012.

11. Waters B, Panicek DM, Lefkowitz RA, et al. Low-grade myxofibrosarcoma: CT and MRI patterns in recurrent disease. AJR Am J Roentgenol 188:W193-W198, 2007.

12. Frassica FJ, Chang BW, Ma LD, et al. Soft tissue sarcomas: General features, evaluation, imaging, biopsy and treatment. Curr Orthopaedicts 11:105-113, 1997.

13. Capra GG, Carbone PN, and Mullin DP. Paranasal sinus mucocele. Head Neck Pathol 6:369-372, 2012.

14. Kaya M, Wada T, Nagoya S, et al. MRI and histological evaluation of the infiltrative growth pattern of myxofibrosarcoma. Skeletal Radiol 37:1085-1090, 2008.

15. Norval EJ, and Raubenheimer EJ. Myxofibrosarcoma arising in the maxillary sinus: A case report with a review of the ultrastructural findings and differential diagnoses. J Maxillofac Oral Surg 10:334-339, 2011.

16. Sanfilippo R, Miceli R, Grosso F, et al. Myxofibrosarcoma: Prognostic factors and survival in a series of patients treated at a single institution. Ann Surg Oncol 18:720-725, 2011.

17. Cante D, Franco P, Sciacero P, et al. Combined chemoradiation for head and neck region myxofibrosarcoma of the maxillary sinus. Tumori 99:e80-e83, 2013. 